Pacific Journal of Mathematics

ON ACCRETIVE OPERATORS ON

SerpeNs AND LOThar HeTZE 


\title{
ON ACCRETIVE OPERATORS ON $l_{n}^{\infty}$
}

\author{
H. BERENS AND L. HeTzelT
}

To Professor H. G. Tillmann on the occasion of his 60 th birthday

\begin{abstract}
It is the object of the paper to discuss the result of Crandall and Liggett on $m$-accretive operators in $l_{n}^{p}$ in greater detail.
\end{abstract}

1. Introduction. By Zorn's Lemma any accretive operator has a maximally accretive extension. In this respect G. J. Minty [12] proved in 1962 that if $H$ is a Hilbert space the accretive (= monotone) operator $A \subset H \times H$ is maximally accretive exactly when there exists a $\lambda \in \mathbf{R}^{+}$ such that (and consequently for all $\lambda \in \mathbf{R}^{+}$)

$$
\forall y \in H \quad x+\lambda a=y \quad \text { has a (unique) solution in } A \text {, }
$$

i.e., $I+\lambda A$ defines a bijection of $A$ onto $H$. In this case, $A$ is said to be $m$-accretive. See the following section for the relevant definitions and notations.

In contrast to Minty's result, M. G. Crandall and T. W. Liggett [6] showed in 1971 that for $l_{n}^{p}, n \in \mathbf{N}, \geq 2$ and $1<p \leq \infty$, the class of $m$-accretive operators coincides with the class of maximally accretive ones exactly when $p=2$, or $\infty$. In particular, we want to reprove their

(1.2) TheOReM (Crandall-Liggett). In $l_{n}^{\infty}, n \in \mathbf{N}$, the class of maximally accretive operators coincides with the class of $m$-accretive ones.

In the following section we do the necessary preliminary work, while $\S 3$ is devoted to the proof of the theorem. In $\$ 4$ we comment on the theorem. Section 5, finally, deals with the domain and range of $m$-accretive operators.

We would like to thank Professor K. Donner for the many valuable discussions we had on this and related subjects.

2. Definitions, Notations, and Preliminaries. Let $X$ be a finite dimensional, real, normed vector space with $\|\cdot\|$. It's elements are denoted by $x, y, z, \ldots$ For a subset $K$ of $X, \bar{K}, \stackrel{\circ}{K}$, and $\partial K$ denote its closure, interior, and boundary, respectively. The open ball centered at $x$ with radius $r \in \mathbf{R}^{+}$is denoted by $b_{r}(x)$. 
The semi-inner product $\langle\cdot, \cdot\rangle_{s}: X \times X \rightarrow \mathbf{R}$ is defined by

$$
\langle y, x\rangle_{s}:=\lim _{t \rightarrow 0+} \frac{\|x+t y\|^{2}-\|x\|^{2}}{2 t}, \quad(y, x) \in X \times X .
$$

If $F$ denotes the duality map on $X$ into its dual $-F$ is the subdifferential of $\|\cdot\|^{2} / 2: X \rightarrow \mathbf{R}$-then

$$
\langle y, x\rangle_{s}=\max \{\langle y, w\rangle: w \in F(x)\} .
$$

It follows that $\langle\cdot, \cdot\rangle_{s}$ is upper semi-continuous on $X \times X$. Furthermore, if $X$ is strictly convex, then $F(x) \cap F\left(x^{\prime}\right)=\varnothing$ whenever $x \neq x^{\prime}$. If $X$ is smooth, then $F$ is single-valued and $\langle y, x\rangle_{s}$ is just the derivative of $\|\cdot\|^{2} / 2$ at $x$ in the direction $y$. If, in particular, $X$ is an inner product space, the semi-inner product reduces to the inner product on $X \times X$. For $l_{n}^{p}, 1<p \leq \infty$

$$
\langle y, x\rangle_{s}=\left\{\begin{array}{l}
\sum_{i=1}^{n} y_{i} \operatorname{sgn} x_{i}\left|x_{i}\right|^{p-1} /\|x\|_{p}^{p-2}, \quad 1<p<\infty, \\
\max \left\{y_{i} x_{i}: 1 \leq i \leq n,\left|x_{i}\right|=\|x\|_{\infty}\right\}, \quad p=\infty, \\
x, y \in l_{n}^{p}, x \neq 0 .
\end{array}\right.
$$

For $x, y \in X, x \neq y$,

$$
C(y, x):=\bigcup_{\lambda>0} b_{\lambda\|x-y\|}(y+\lambda(x-y))
$$

denotes the cone of decrease of $x$ with vertex at $y . C(y, x)$ is the open tangential cone of $b_{\|x-y\|}(x)$ at $y$. With use of the semi-inner product the cone of decrease can be rewritten as

$$
C(y, x)=\left\{z \in X:\langle y-z, x-y\rangle_{s}<0\right\} .
$$

If $x=y C(y, x)$ is defined to be the empty set.

The set of intermediate points between $x$ and $y$ in $X$ is defined by

$$
Z(x, y):=\{z \in X:\|x-z\|+\|z-y\|=\|x-y\|\} .
$$

A $\|\cdot\|$-segment in $X$ is a curve whose length equals the distance of its endpoints.

We say that $K \subset X$ is $\|\cdot\|$-convex, if

$$
\forall k^{\prime}, k^{\prime \prime} \in K \exists k \in K, k \neq k^{\prime}, k^{\prime \prime} \ni k \in Z\left(k^{\prime}, k^{\prime \prime}\right) \text {. }
$$

If $K$ is a closed subset of $X$ there is a useful characterization of $\|\cdot\|$-convexity, see [5, p. 29].

(2.1) LeMMA. Let $K$ be a closed set in $X . K$ is $\|\cdot\|$-convex exactly when every two points in $K$ can be joint by $a\|\cdot\|$-segment completely contained in K. 
If $X$ is strictly convex, then $\|\cdot\|$-convexity reduces to the classical notion of convexity. For $l_{n}^{1}$ - this is the case we are particularly interested in-the intermediate points of $x$ and $y$ are given by the parallelepiped spanned by $x$ and $y$. And $\left[0,\|y-x\|_{1}\right] \ni t \mapsto z(t)$ defines a $\|\cdot\|_{1}$-segment joining $x$ and $y$, if it is a continuous curve with $x$ and $y$ as endpoints which is monotone in each component $z_{i}(t), 0 \leq t \leq\|y-x\|_{1}$.

A set-valued map $A$ from $X$ into itself is called an operator on $X$. It is convenient to identify $A$ with its graph in $X \times X . D(A):=\{x \in X$ : $(x, a) \in A\}$ and $R(A):=\{a \in X:(x, a) \in A\}$ denote the domain and range of $A$, respectively. $A^{-1}:=\{(a, x) \in X \times X:(x, a) \in A\}$. If $A^{1}$ and $A^{2}$ are two operators on $X, A^{1}+A^{2}:=\left\{\left(x, a^{1}+a^{2}\right) \in X \times X:\left(x, a^{1}\right) \in\right.$ $A^{1}$ and $\left.\left(x, a^{2}\right) \in A^{2}\right\}$, and for $\lambda \in \mathbf{R}^{+} \lambda A:=\{(x, \lambda a) \in X \times X:(x, a)$ $\in A\}$.

The operator $A$ is said to be accretive if

$$
\forall(x, a),\left(x^{\prime}, a^{\prime}\right) \in A \quad 0 \leq\left\langle a-a^{\prime}, x-x^{\prime}\right\rangle_{s} .
$$

This is equivalent to the fact that

$$
\forall \lambda \in \mathbf{R}^{+} \quad(I+\lambda A)^{-1} \text { defines a contraction from } X \text { to itself, }
$$
i.e.,

$$
\begin{aligned}
\forall(x, a),\left(x^{\prime}, a^{\prime}\right) & \in A \\
\left\|x-x^{\prime}\right\| & \leq\left\|(x+\lambda a)-\left(x^{\prime}+\lambda a^{\prime}\right)\right\|, \quad \forall \lambda \in \mathbf{R}^{+} .
\end{aligned}
$$

$A$ is said to be $m$-accretive if $A$ is accretive and if for all $\lambda \in \mathbf{R}^{+} I+\lambda A$ is surjective. (It follows trivially from the accretiveness of $A$ that $I+\lambda A$ is injective, thus if $A$ is $m$-accretive for each $\lambda \in \mathbf{R}^{+} I+\lambda A$ is bijective.) An $m$-accretive operator on $X$ is a maximal element within the class of accretive operators on $X$ ordered by inclusion.

If $X$ is an inner product space it is common to speak of monotone operators instead of accretive ones.

3. Proof of the Theorem. The key to our proof is

(3.1) ThEOREM. Let $A$ be a finite accretive set in $l_{n}^{\infty} \times l_{n}^{\infty}$, then

$$
\forall x \in l_{n}^{\infty} B_{A}(x):=\left\{a \in l_{n}^{\infty}:\left\langle a^{\prime}-a, x^{\prime}-x\right\rangle_{s} \geq 0 \forall\left(x^{\prime}, a^{\prime}\right) \in A\right\}
$$

is nonempty, closed, and $\|\cdot\|_{1}$-convex. If $Q_{A}$ denotes the smallest closed parallelepiped $^{1}$ in $l_{n}^{\infty}$ containing $R(A)$, then even $B_{A}^{Q}(x):=Q_{A} \cap B_{A}(x)$ is nonempty, compact, and $\|\cdot\|_{1}$-convex.

${ }^{1}$ The faces of $Q_{A}$ are assumed to be parallel to the main axes. 
Let $A:=\left\{\left(x^{1}, a^{1}\right), \ldots,\left(x^{m}, a^{m}\right)\right\}, m \in \mathbf{N}$, and let $x \in l_{n}^{\infty}$. Setting

$$
C^{j}:=C\left(a^{j}, a^{j}+x^{j}-x\right), \quad 1 \leq j \leq m,
$$

Theorem 3.1 claims that

$$
B_{A}(x)=\bigcap_{j=1}^{m} \mathbf{C} C^{j} \text { is } \neq \varnothing, \text { closed and }\|\cdot\|_{1} \text {-convex. }
$$

Let us introduce some further notation

$$
I_{ \pm}^{j}:=\left\{1 \leq i \leq n: x_{i}^{j}-x_{i}= \pm\left\|x^{j}-x\right\|_{\infty}\right\} \quad \text { and } \quad I^{j}:=I_{+}^{j} \cup I_{-}^{j} .
$$

To prove Theorem 3.1 we start with

(3.2) LEMMA.

$$
\forall 1 \leq j, l \leq m \quad C^{j} \cap C^{l} \text { is a cone. }
$$

Proof. It is an easy exercise to verify that

$$
\begin{aligned}
C^{j} \cap C^{l}= & \left\{z \in l_{n}^{\infty}: z_{i}>a_{i}^{j}, i \in I_{+}^{j} \backslash I^{l}\right\} \\
& \cap\left\{z \in l_{n}^{\infty}: z_{i}<a_{i}^{j}, i \in I_{-}^{j} \backslash I^{l}\right\} \\
& \cap\left\{z \in l_{n}^{\infty}: z_{i}>a_{i}^{l}, i \in I_{+}^{l} \backslash I^{j}\right\} \\
& \cap\left\{z \in l_{n}^{\infty}: z_{i}<a_{i}^{l}, i \in I_{-}^{l} \backslash I^{j}\right\} \\
& \cap\left\{z \in l_{n}^{\infty}: z_{i}>\max \left(a_{i}^{j}, a_{i}^{l}\right), i \in I_{+}^{j} \cap I_{+}^{l}\right\} \\
& \cap\left\{z \in l_{n}^{\infty}: z_{i}<\min \left(a_{i}^{j}, a_{i}^{l}\right), i \in I_{-}^{j} \cap I_{-}^{l}\right\} \\
& \cap\left\{z \in l_{n}^{\infty}: a_{i}^{j}<z_{i}<a_{i}^{l}, i \in I_{+}^{j} \cap I_{-}^{l}\right\} \\
& \cap\left\{z \in l_{n}^{\infty}: a_{i}^{l}<z_{i}<a_{i}^{j}, i \in I_{-}^{j} \cap I_{+}^{l}\right\} .
\end{aligned}
$$

Hence, if $C^{j} \cap C^{l} \neq \varnothing$

$$
C^{j} \cap C^{l} \text { is a cone iff } I_{+}^{j} \cap I_{-}^{l}=\varnothing \text { and } I_{-}^{j} \cap I_{+}^{l}=\varnothing .
$$

But if $i \in I_{+}^{j} \cap I_{-}^{l}$, then

$$
\begin{aligned}
x_{i}^{j}-x_{i}^{l} & =\left(x_{i}^{j}-x_{i}\right)-\left(x_{i}^{l}-x_{i}\right) \\
& =\left\|x^{j}-x\right\|_{\infty}+\left\|x^{l}-x\right\|_{\infty}=\left\|x^{j}-x^{l}\right\|_{\infty},
\end{aligned}
$$

and consequently,

$$
\left(a_{i}^{j}-a_{i}^{l}\right)\left(x_{i}^{j}-x_{i}^{l}\right)=\left\langle a^{j}-a^{l}, x^{j}-x^{l}\right\rangle_{s} \geq 0
$$


implies $a_{i}^{l} \leq a_{i}^{j}$, or $C^{j} \cap C^{l}=\varnothing$. Similarly, if $i \in I_{-}^{j} \cap I_{+}^{l}$, then $x_{i}^{j}-x_{i}^{l}$ $=-\left\|x^{j}-x^{l}\right\|_{\infty}$, by (3.3) $a_{i}^{j} \leq a_{i}^{l}$ which again implies $C^{j} \cap C^{l}=\varnothing$.

Proof of Theorem 3.1. We prove the theorem by induction on $m=$ $\operatorname{card}(A)$. For $m=1$ there is nothing to prove. Let us assume that for all accretive sets of cardinality $m-1$ the theorem holds true.

If $x=x^{j}$ for some $1 \leq j \leq m$, we are done. Let us therefore assume that $x \neq x^{j}, 1 \leq j \leq m$.

By the induction hypothesis

$$
\bigcap_{j=1}^{m-1} \mathbf{C} C^{j} \neq \varnothing, \quad \text { closed, and }\|\cdot\|_{1} \text {-convex. }
$$

Obviously, $B_{A}(x)$ is closed. First, we prove that it is nonempty. In contrast to our claim, let us assume that $B_{A}(x)=\varnothing$. Consequently,

$$
\mathbf{C} C^{m} \subseteq \bigcup_{j=1}^{m-1} C^{j}
$$

W.1.o.g. let $I^{m}=\{1,2, \ldots, s\}, 1 \leq s \leq n$. We define

$$
J_{0}:=\left\{1 \leq j \leq m-1: C^{j} \cap S^{m} \neq \varnothing\right\},
$$

where $S^{m}=\left\{b \in l_{n}^{\infty}: b_{i}=a_{i}^{m}, i \in I^{m}\right\}$ is the vertex set of $C^{m}$. Since $S^{m} \subset \mathbf{C} C^{m}$, by (3.5) $J_{0} \neq \varnothing$. We claim,

$$
\exists j_{0} \in J_{0} \ni I^{j_{0}} \subset I^{m} \text {. }
$$

If not, then $J_{0}=\bigcup_{i=s+1}^{n} D^{i}$, where $D^{i}=D_{+}^{i} \cup D_{-}^{i}$ and $D_{ \pm}^{i}=\left\{j \in J_{0}\right.$ : $\left.i \in I_{ \pm}^{j}\right\}$. We select a sub-manifold $S_{0}^{m} \subset S^{m}$ subject to the following restrictions. For $s+1 \leq i \leq n$ and $D^{i} \neq \varnothing$ we set $b_{i}^{0}=\min \left\{a_{i}^{j}: j \in\right.$ $\left.D_{+}^{i}\right\}$, in case $D_{+}^{i} \neq \varnothing$, otherwise $=\max \left\{a_{i}^{j}: j \in D_{-}^{i}\right\}$. In the first case, if $D_{-}^{i} \neq \varnothing$ too, for all $l \in D_{-}^{i} a_{i}^{l} \leq b_{i}^{0}$, for, if for some $j \in D_{+}^{i}$ and some $l \in D_{-}^{i} a_{i}^{j}<a_{i}^{l}$, then $C^{j} \cap C^{l} \neq \varnothing$ while $I_{+}^{j} \cap I_{-}^{l} \neq \varnothing$, contradicting Lemma 3.2.

We define $S_{0}^{m}:=\left\{b \in S^{m}: b_{i}=b_{i}^{0}, s+1 \leq i \leq n, \quad D_{i} \neq \varnothing\right\}$. By construction $S_{0}^{m} \subset \mathbf{C} C^{j}$ for all $j \in D^{i}, s+1 \leq i \leq n$, hence for all $j \in J_{0}$. Trivially, $S_{0}^{m} \subset \mathbf{C} C^{m}$ and $S_{0}^{m} \subset \mathbf{C} C^{j}$ for all $1 \leq j \leq m-1$ not belonging to $J_{0}$. Thus $S_{0}^{m} \subset B_{A}(x)$, which contradicts the assumption that $B_{A}(x)=\varnothing$.

Hence there exists an index $j_{0} \in J_{0}$ such that $I^{j_{0}} \subseteq I^{m}$. Since $C^{j_{0}} \cap$ $C^{m} \neq \varnothing$, by use of Lemma $3.2 I_{+}^{j_{0}} \cap I_{-}^{m}$ and $I_{-}^{j_{0}} \cap I_{+}^{m}$ are empty, i.e., $I_{+}^{j_{0}} \subseteq I_{+}^{m}$ and $I_{-}^{j_{0}} \subseteq I_{-}^{m}$. But this implies that $C^{m} \subset C^{j_{0}}$, and consequently, $B_{A}(x)=\bigcap_{j=1}^{m-1} \mathbf{C} C^{j}$ which is nonempty by the induction hypothesis (3.4), again in contradiction to our assumption that $B_{A}(x)=\varnothing$. 
To prove that $B_{A}(x)$ is $\|\cdot\|_{1}$-convex, let us assume there exist two points in $B_{A}(x)$ which have no proper intermediate points. W.1.o.g. we may assume that 0 and $a$ are these points where $a_{i}>0,1 \leq i \leq s$ and $a_{i}=0, s+1 \leq i \leq n$, for some $1 \leq s \leq n$. Hence

$$
Z(0, a) \cap B_{A}(x)=\left(Z(0, a) \cap \mathbf{C} C^{m}\right) \cap\left(Z(0, a) \cap \bigcap_{j=1}^{m-1} \mathbf{C} C^{j}\right)=\{0, a\} .
$$

Since by the induction hypothesis (3.4) $\bigcap_{j=1}^{m-1} \mathbf{C} C^{j}$ is $\|\cdot\|_{1}$-convex, it follows that

$$
\{0, a\} \subset \partial C^{m} \text { and } Z(0, a) \cap C^{m} \neq \varnothing
$$

-indeed, any $\|\cdot\|_{1}$-segment in $\bigcap_{j=1}^{m-1} \mathbf{C} C^{j}$ connecting 0 and $a$ belongs to $C^{m}$ except for the two endpoints. Consequently, there are two indices $i_{0}$ and $i_{a}, 1 \leq i_{0}, i_{a} \leq s, i_{0} \neq i_{a}$ (by use of Lemma 3.2) such that $i_{0} \in I_{+}^{m}$ and $i_{a} \in I_{-}^{m}$. W.1.o.g. we may further assume that $i_{0}=1$.

Consider the point

$$
b_{\varepsilon}:=\left(\varepsilon, a_{2}, \ldots, a_{n}\right) \text { in } Z(0, a), \quad 0<\varepsilon<a_{1} .
$$

It is a proper intermediate point of 0 and $a$ located on $\partial C^{m}$. Since by assumption $Z(0, a) \cap B_{A}(x)=\{0, a\}$, there exists an index $j, 1 \leq j \leq$ $m-1$, such that $b_{\varepsilon} \in C^{j}$. Obviously, $C^{m} \cap C^{j} \neq \varnothing$. By Lemma 3.2 $I_{+}^{m} \cap I_{-}^{j}$ and $I_{-}^{m} \cap I_{+}^{j}$ are empty.

If $1 \notin I^{j}$, then $a \in C^{j}$, contradicting $a \in B_{A}(x)$. Hence $1 \in I^{j}$. If $1 \in I_{+}^{j}$, then again $a \in C^{j}$, contradicting $a \in B_{A}(x)$. Hence $1 \in I_{-}^{j}$. Since $1 \in I_{+}^{m}$, this is in contradiction to $I_{+}^{m} \cap I_{-}^{j}=\varnothing$. Thus any given two points in $B_{A}(x)$ have proper intermediate points in $B_{A}(x)$ with respect to the $\|\cdot\|_{1}$-norm.

Let $Q_{A}$ be the smallest closed parallelepiped containing $R(A)$. To prove that even

$$
Q_{A} \cap B_{A}(x) \text { is nonempty, compact, and }\|\cdot\|_{1} \text {-convex, }
$$

we extend $A$ via

$$
A_{\mathrm{ext}}:=A \cup \bigcup_{i=1}^{n}\left\{\left(x^{i, \pm}, a^{i, \pm}\right) \in l_{n}^{\infty} \times l_{n}^{\infty}\right\},
$$

where for each $1 \leq i \leq n$

$$
x^{i, \pm}:=x \pm r e_{i},
$$

$e_{i}$ being the $i$ th unit vector in $l_{n}^{\infty}$ and $r=2 \max \left\{\left\|x-x^{j}\right\|_{\infty}: 1 \leq j \leq m\right\}$, and

$$
a^{i, \pm} \in Q_{A}, \quad a_{i}^{i,+}:=\max _{1 \leq j \leq m} a_{i}^{j} \text { and } a_{i}^{i,-}:=\min _{1 \leq j \leq m} a_{i}^{j} .
$$


By definition, $A_{\text {ext }}$ is accretive and $B_{A_{\text {ext }}}(x)=Q_{A} \cap B_{A}(x)$.

(3.7) Proposition. Let $A$ be a maximally accretive operator on $l_{n}^{\infty}$. $\forall x \in D(A) A(x)$ is closed and $\|\cdot\|_{1}$-convex.

Proof. By the maximality of $A$ and by the u. semi-continuity of the semi-inner product for each $x \in D(A) A(x)$ is closed.

To see that $A(x)$ is $\|\cdot\|_{1}$-convex, let $\Gamma$ be the net of all finite subsets $A_{\gamma}=\left\{\left(x^{1}, a^{1}\right), \ldots,\left(x^{m_{\gamma}}, a^{m_{\gamma}}\right)\right\}, m_{\gamma} \in \mathbf{N}$, of $A$ subject to the restriction that $x^{j} \neq x, 1 \leq j \leq m_{\gamma} ; \gamma<\gamma^{\prime}$ if $A_{\gamma} \subseteq A_{\gamma^{\prime}}$. Clearly,

$$
\begin{aligned}
A(x) & \subseteq B_{A}(x):=\bigcap_{\substack{\left(x^{\prime}, a^{\prime}\right) \in A \\
x^{\prime} \neq x}}\left\{b \in l_{n}^{\infty}:\left\langle a^{\prime}-b, x^{\prime}-x\right\rangle_{s} \geq 0\right\} \\
& =\lim _{\gamma \in \Gamma} B_{A_{\gamma}}(x) .
\end{aligned}
$$

We show that $B_{A}(x)$ is $\|\cdot\|_{1}$-convex. Indeed, take $b^{\prime}$ and $b^{\prime \prime}$ in $B_{A}(x)$, and for each $\gamma \in \Gamma$ let $\left[0,\left\|b^{\prime \prime}-b^{\prime}\right\|_{1}\right] \ni t \mapsto b_{\gamma}(t)$ be a $\|\cdot\|_{1}$-segment in $B_{A_{\gamma}}(x)$ connecting $b^{\prime}$ and $b^{\prime \prime}$. By definition, $b_{\gamma}$ is Lipschitz-continuous, i.e.,

$$
\forall 0 \leq t^{\prime}<t^{\prime \prime} \leq\left\|b^{\prime \prime}-b^{\prime}\right\|_{1}, \quad\left\|b_{\gamma}\left(t^{\prime \prime}\right)-b_{\gamma}\left(t^{\prime}\right)\right\|_{1}=t^{\prime \prime}-t^{\prime} .
$$

By the theorem of Arzela-Ascoli, any accumulation point of the net $\left\{b_{\gamma}\right.$ : $\gamma \in \Gamma\}$ defines a $\|\cdot\|_{1}$-segment in $B_{A}(x)$ connecting $b^{\prime}$ and $b^{\prime \prime}$.

By the maximality of $A$, however, $A(x)=B_{A}(x)$.

(3.8) Proposition. Let $A$ be an accretive operator with $R(A) \subseteq Q$, a compact parallelepiped.

If $A$ is maximal with respect to all accretive operators with range in $Q$, then $\forall x \in l_{n}^{\infty} A(x)$ is nonempty, compact, and $\|\cdot\|_{1}$-convex.

Proof. By use of the notation introduced in the proofs of Theorem 3.1 and Proposition 3.7, for each $x \in l_{n}^{\infty}$

$$
\begin{aligned}
A(x) & \subseteq B_{A}^{Q}(x):=\bigcap_{\substack{\left(x^{\prime}, a^{\prime}\right) \in A \\
x^{\prime} \neq x}}\left\{b \in Q:\left\langle a^{\prime}-b, x^{\prime}-x\right\rangle_{s} \geq 0\right\} \\
& =\lim _{\gamma \in \Gamma} B_{A_{\gamma}}^{Q}(x) .
\end{aligned}
$$

By Theorem 3.1 for each $\gamma \in \Gamma B_{A_{\gamma}}^{Q}(x)$ is nonempty, compact and $\|\cdot\|_{1}$ convex, and so is $B_{A}^{Q}(x)$ by a compactness argument. The maximality of $A$ again implies that for each $x A(x)=B_{A}^{Q}(x)$. 
Next we want to prove that under the assumptions of Proposition 3.8 an accretive operator is $m$-accretive. The proof rests upon a theorem of surjectivity for set-valued mappings (J. M. Lasry and R. Robert [11, Corollaire 1.18, p. 98]): Let $\Gamma: \mathbf{R}^{n} \rightarrow \mathbf{R}^{n}$ be an upper semi-continuous, compact-valued, acyclic mapping. If $\Gamma$ is coercive, then it is surjective.-For each $x \in \mathbf{R}^{n}$, let $\gamma(x)=\min \{\langle y, x\rangle: y \in \Gamma(x)\} . \Gamma$ is said to be coercive if $\gamma(x) /\|x\| \rightarrow \infty$ when $\|x\| \rightarrow \infty$.

Let $A$ be an accretive operator on $l_{n}^{\infty}$ satisfying the assumptions of Proposition 3.8, and let $\lambda \in \mathbf{R}^{+}$be fixed. By maximality $I+\lambda A$ is upper semi-continuous, and Proposition 3.8 assures that

$$
\forall x \in l_{n}^{\infty} \quad A(x) \neq \varnothing, \text { compact, and }\|\cdot\|_{1} \text {-convex. }
$$

In [1] the authors proved

(3.9) LeMMA. A nonempty, compact, $\|\cdot\|_{1}$-convex set in $l_{n}^{\infty}$ is a $R_{\delta}$-set, i.e., it is the intersection of a decreasing sequence of compact sets which are contractible in itself.

By J. M. Lasry and R. Robert [11, Proposition 2.1, p. 110] $R_{\delta}$-sets in $\mathbf{R}^{n}$ are acyclic (in the sense of Čech-cohomology).

Since $R(A)$ is contained in the compact parallelepiped $Q, I+\lambda A$ is trivially coercive. Thus the conditions of the theorem of surjectivity are fulfilled, giving

(3.10) Proposition. Let $A$ be an accretive operator on $l_{n}^{\infty}$ with $R(A) \subseteq$ $Q$, a compact parallelepiped.

If $A$ is maximal with respect to all accretive operators with range in $Q$, then $A$ is $m$-accretive.

REMARK. To prove Proposition 3.10, instead of the theorem of surjectivity due to Lasry-Robert we may use a set-valued version of the theorem of the invariance of domain (A. Granas and J. W. Jaworowski [8]): Let $U$ be an open subset of $\mathbf{R}^{n}$ and let $\phi: U \rightarrow \mathbf{R}^{n}$ be an upper semi-continuous, compact-valued, acyclic mapping such that $\Phi(x) \cap \Phi\left(x^{\prime}\right)=\varnothing$ whenever $x \neq x^{\prime}$, then $\Phi(U)$ is open. Indeed, for each $\lambda \in \mathbf{R}^{+} I+\lambda A$ satisfies the assumptions of the theorem.

Now we are ready to prove the Theorem.

Let $A$ be a maximally accretive operator on $l_{n}^{\infty}$. W.l.o.g. let $(0,0) \in A$. 
For each $m \in \mathbf{N}$, we define the restriction $A^{m}=\left\{(x, a) \in A:\|a\|_{\infty}\right.$ $\leq m\}$ of $A$. Let $B^{m}$ be a maximal extension of $A^{m}$ subject to the restriction that for all $(x, b) \in B^{m}\|b\|_{\infty} \leq m$. By Proposition $3.10 B^{m}$ is $m$-accretive.

Let $\lambda \in \mathbf{R}^{+}$be fixed. Assume that $I+\lambda A$ is not surjective. We take a point in $l_{n}^{\infty}$, say $y$, contained in the complement of $(I+\lambda A)\left(l_{n}^{\infty}\right)$. Since for each $m \in \mathbf{N} B^{m}$ is $m$-accretive

$$
y=x^{m}+\lambda b^{m} \quad \text { for some }\left(x^{m}, b^{m}\right) \in B^{m} .
$$

Since $\left\|x^{m}\right\|_{\infty} \leq\|y\|_{\infty}$, there exists a convergent subsequence, say $\lim _{j} x^{m_{j}}$ $=x$. Consequently, $\lim _{j} b^{m_{j}}=(y-x) / \lambda=: b$. Take a pair $\left(x^{\prime}, a^{\prime}\right) \in A$. For $m_{j} \geq\left\|a^{\prime}\right\|_{\infty}\left(x^{\prime}, a^{\prime}\right) \in B^{m_{j}}$ and by the $u$. semi-continuity of the semi-inner product

$$
0 \leq \varlimsup_{\lim _{j}}\left\langle b^{m_{j}}-a^{\prime}, x^{m_{j}}-x^{\prime}\right\rangle_{s} \leq\left\langle b-a^{\prime}, x-x^{\prime}\right\rangle_{s} .
$$

By construction, $(x, b) \notin A$. Hence $A \cup(x, b)$ properly extends $A$, which contradicts its maximality.

REMARK. The proof of Crandall and Liggett runs as follows:

Let $A$ be a maximally accretive operator on $l_{n}^{\infty}$.

Fix an element $y \in l_{n}^{\infty}$. They claim that $y \in R(I+A)(\lambda$ is set to be equal to 1). To prove their claim they define

$$
\forall\left(x^{\prime}, a^{\prime}\right) \in A \quad V\left(x^{\prime}, a^{\prime}\right)=\left\{z \in l_{n}^{\infty}:\left\langle y-z-a^{\prime}, z-x^{\prime}\right\rangle_{s} \geq 0\right\} .
$$

If $\bigcap\left\{V\left(x^{\prime}, a^{\prime}\right):\left(x^{\prime}, a^{\prime}\right) \in A\right\}$ is not empty, say $z^{0}$ belongs to the intersection, then by the maximality of $A\left(z^{0}, y-z^{0}\right) \in A$, giving $y=z^{0}+$ $\left(y-z^{0}\right)$. For each $\left(x^{\prime}, a^{\prime}\right) \in A V\left(x^{\prime}, a^{\prime}\right)$ is nonempty and compact. Thus it remains to verify that $\left\{V\left(x^{\prime}, a^{\prime}\right):\left(x^{\prime}, a^{\prime}\right) \in A\right\}$ has the finite intersection property.

To do this, let $B=\left\{\left(x^{1}, b^{1}\right), \ldots,\left(x^{m}, b^{m}\right)\right\}, m \in \mathbf{N}$ be a finite accretive operator and let $D_{B}$ be the smallest closed parallelepiped which contains $y-b^{j}, 1 \leq j \leq m$. Define $T: D_{B} \rightarrow D_{B}$ by

$$
D_{B} \ni x \mapsto T(x)=\left\{z \in D_{B}:\left\langle(y-z)-b^{j}, x-x^{j}\right\rangle_{s} \geq 0,1 \leq j \leq m\right\} \text {. }
$$

The crucial part of their proof is the verification of the following

(3.11) LEMMA.

$\forall x \in D_{B} \quad T(x) \neq \varnothing$, compact, and contractible in itself. 
Obviously, $T$ is upper semi-continuous. By the fixed point theorem of Eilenberg and Montgomery $T$ has a fixed point in $D_{B}$. This proves that $\left\{V\left(x^{\prime}, a^{\prime}\right):\left(x^{\prime}, a^{\prime}\right) \in A\right\}$ has the finite intersection property.

4. Remarks about the Theorem. The counterpart to Theorem 3.1 in $\mathbf{R}^{n}$ was formulated and proved by G. J. Minty [13] in 1962.

(4.1) Theorem. Let $A$ be a finite monotone set in $\mathbf{R}^{n} \times \mathbf{R}^{n}$. Then $\forall x \in \mathbf{R}^{n} B_{A}(x):=\left\{a \in \mathbf{R}^{n}:\left\langle a^{\prime}-a, x^{\prime}-x\right\rangle \geq 0 \forall\left(x^{\prime}, a^{\prime}\right) \in A\right\}$ is nonempty, closed and convex.

If $Q_{A}=\operatorname{co}\left\{a^{\prime} \in \mathbf{R}^{n}:\left(x^{\prime}, a^{\prime}\right) \in A\right\}$, then even the intersection $Q_{A} \cap$ $B_{A}(x)$ is nonempty, compact, and convex.

To be more precise than above, the first statement of the theorem is due to Minty, its extension was proved by $\mathrm{H}$. Debrunner and P. Flor [7] in 1964, and the proof of Minty's maximality theorem given in H. Brezis [3, Theorem 2.1, p. 23f] is based on their extension.

For $l_{2}^{p}, 1<p<\infty, \quad p \neq 2$, Crandall and Liggett considered the following operator $A$ : Let $\left\{e_{1}, e_{2}\right\}$ be the natural basis in $l_{2}^{p}$, and let

$$
A:=\left\{(0,0),\left(e_{1}, e_{2}\right),\left(e_{2},-e_{1}\right)\right\} .
$$

They pointed out that no maximally accretive extension of $A$ on $l_{2}^{p}$ is defined on the triangle $\left\{\left(\xi_{1}, \xi_{2}\right) \in l_{2}^{p}: 0<\xi_{1}<\xi_{2}, \xi_{1}+\xi_{2}<1\right\}$ if $1<p$ $<2$, respectively on the triangle $\left\{\left(\xi_{1}, \xi_{2}\right) \in l_{2}^{p}: 0<\xi_{2}<\xi_{1}, \xi_{1}+\xi_{2}<1\right\}$ if $2<p<\infty$, in contrast to the fact that the closure of an $m$-accretive operator on $l_{2}^{p}$ is convex, see Theorem 5.1 below.

In [2] the authors extended their result as follows:

(4.2) For the plane endowed with a strictly convex and smooth norm the class of maximally accretive operators coincides with the class of $m$-accretive ones exactly when the norm generates an inner product.

The stronger statement: In the normed plane the two classes coincide exactly when the unit ball is either an ellipse or a parallelogram, as well as the obvious extension for the $n$-space, are still open, see also the comments of Crandall and Liggett, loc. cit., on this subject.

We want to conclude the section with two statements on maximally accretive operators on the normed plane, which are in the vein of our paper.

Via the quadratic and skew-symmetric form

$$
S(x, y):=\eta_{1} \xi_{2}-\xi_{1} \eta_{2} \quad x=\left(\xi_{1}, \xi_{2}\right) \text { and } y=\left(\eta_{1}, \eta_{2}\right)
$$


we define the so-called dual *norm

$$
\forall x \quad\|x\|^{*}:=\sup \{S(x, y):\|y\| \leq 1\} .
$$

The unit ball of the plane w.r.t. the dual ${ }^{*}$ norm is just the unit ball w.r.t. the dual norm rotated by $90^{\circ}$, and $\|\cdot\|^{* *}=\|\cdot\|$. The following lemma, due to $\mathrm{H}$. Busemann, if of interest in connection with accretiveness on the plane.

(4.3) LEMMA.

$$
\forall x\langle y, x\rangle_{s} \geq 0 \Leftrightarrow\langle x, y\rangle_{s^{*}} \geq 0
$$

We have

(4.4) Let $A$ be a maximally accretive operator on the plane.

$$
\forall x \in D(A) \quad A(x) \text { is closed and }\|\cdot\|^{*} \text {-convex. }
$$

(4.5) Let $A$ be a maximally accretive operator on the plane.

If $A$ is defined on the whole plane then $A$ is $m$-accretive.

We do not want to give formal proofs of the two propositions. The first one is not difficult to verify, while the second one follows from the fact that under the assumptions of the proposition $A$ is upper semi-continuous and

$\forall x A(x)$ is nonempty, compact, and contractible in itself.

5. On the domain and the range of an $m$-accretive operator. In the following $A$ is assumed to be $m$-accretive on $X$. Hence for each $\lambda$ the Yosida-resolvent $J_{\lambda}:=(I+\lambda A)^{-1}$ defines a contraction on $X$.

Following H. Brezis [3, Theorem 2.2] and R. C. Bruck [4] it is not difficult to prove

(5.1) TheOREM. Let $A$ be $m$-accretive on $X$.

$$
\forall y \in X \quad \exists x \in \overline{D(A)} \ni 0 \leq\left\langle y-x, x-x^{\prime}\right\rangle_{s} \quad \forall x^{\prime} \in \overline{D(A)} \text {. }
$$

Moreover, $\overline{D(A)}$ is $\|\cdot\|$-convex.

If $X$ is strictly convex, $\overline{D(A)}$ is just convex. If $X$ is smooth, then $\overline{D(A)}$ is the range of a uniquely defined contractive projection, say $P_{\overline{D(A)}}$, and

$$
\forall y \in X \quad \lim _{\lambda \rightarrow 0+} J_{\lambda} y=P_{\overline{D(A)}} y=x .
$$

Proof. Take an element $y \in X$.

$$
\forall \lambda \in \mathbf{R}^{+} \quad \exists\left(x_{\lambda}, a_{\lambda}\right) \in A \ni x_{\lambda}+\lambda a_{\lambda}=y .
$$


By the accretiveness of $A$

$$
0 \leq\left\langle a_{\lambda}-a^{\prime}, x_{\lambda}-x^{\prime}\right\rangle_{s} \quad \forall\left(x^{\prime}, a^{\prime}\right) \in A,
$$

or, multiplying the inequality by $\lambda$ and replacing $\lambda a_{\lambda}$ by $y-x_{\lambda}$,

$$
0 \leq\left\langle y-x_{\lambda}-\lambda a^{\prime}, x_{\lambda}-x^{\prime}\right\rangle_{s} \quad \forall\left(x^{\prime}, a^{\prime}\right) \in A .
$$

On the other hand, $\left\{x_{\lambda}\right\}_{\lambda>0}$ is bounded for $\lambda \rightarrow 0+$-indeed, for each $\left(x^{\prime}, a^{\prime}\right) \in A\left\|x_{\lambda}-x^{\prime}\right\| \leq\left\|y-\left(x^{\prime}+\lambda a^{\prime}\right)\right\|$. Since the semi-inner product is upper semi-continuous, for any accumulation point $x$ of $\left\{x_{\lambda}\right\}_{\lambda>0}$ for $\lambda \rightarrow 0+$,

$$
0 \leq\left\langle y-x, x-x^{\prime}\right\rangle_{s} \quad \forall x^{\prime} \in \overline{D(A)} .
$$

Since $\left\{x_{\lambda}\right\}_{\lambda>0} \subset D(A), x \in \overline{D(A)}$.

The inequality (5.2) implies that

$$
\begin{aligned}
\left\|x-x^{\prime}\right\|^{2} & \leq\left\langle y-x^{\prime}, x-x^{\prime}\right\rangle_{s} \\
& \leq\left\|y-x^{\prime}\right\|\left\|x-x^{\prime}\right\|, \quad \forall x^{\prime} \in \overline{D(A)},
\end{aligned}
$$

from which we easily derive that $\overline{D(A)}$ is $\|\cdot\|$-convex. Indeed, let $x^{\prime}$ and $x^{\prime \prime}$ be elements of $\overline{D(A)}$. If $y=\left(x^{\prime}+x^{\prime \prime}\right) / 2 \in \overline{D(A)}$, we are done. If not, let $x \in \overline{D(A)}$ be such that (5.2) holds. By (5.3),

$$
\left\|x-x^{\prime}\right\| \leq\left\|y-x^{\prime}\right\| \text { and }\left\|x-x^{\prime \prime}\right\| \leq\left\|y-x^{\prime \prime}\right\|,
$$

but $\left\|y-x^{\prime}\right\|=\left\|y-x^{\prime \prime}\right\|=\left\|x^{\prime}-x^{\prime \prime}\right\| / 2$ which implies that $x \neq x^{\prime}, x^{\prime \prime}$ and that

$$
\left\|x^{\prime}-x^{\prime \prime}\right\| \leq\left\|x^{\prime}-x\right\|+\left\|x^{\prime \prime}-x\right\| \leq\left\|x^{\prime}-x^{\prime \prime}\right\|,
$$

$x$ is consequently a proper intermediate point between $x^{\prime}$ and $x^{\prime \prime}$ in $\overline{D(A)}$.

If $x$ is smooth then the semi-inner product is linear in its first variable. Let $y \in X$ and $x_{1}, x_{2} \in D(A)$ be such that

$$
0 \leq\left\langle y-x_{i}, x_{i}-x^{\prime}\right\rangle_{s}
$$

for all $x^{\prime} \in \overline{D(A)}, i=1,2$. It follows that

$$
\begin{aligned}
\left\|x_{1}-x_{2}\right\|^{2} & =\left\langle x_{1}-x_{2}, x_{1}-x_{2}\right\rangle_{s} \\
& =-\left\langle y-x_{1}, x_{1}-x_{2}\right\rangle_{s}-\left\langle y-x_{2}, x_{2}-x_{1}\right\rangle_{s} \leq 0 .
\end{aligned}
$$

Thus for each $y \in X$ there exists at most one element $x \in \overline{D(A)}$ satisfying the inequality in (5.2). By (5.2) there exists such an element, namely,

$$
\lim _{\lambda \rightarrow 0+} J_{\lambda} y=x \text {. }
$$


Let $K$ be a proper closed nonempty subset of $X$. In connection with his study of the fixed point set of contractive mappings, F. E. Browder introduced the so-called approximation region $A(y ; K)$ between $y(\in X)$ and $K$ :

$$
A(y ; K)=\left\{z \in X: 0 \leq\langle y-z, z-k\rangle_{s} \forall k \in K\right\} .
$$

If for each $y \in X, A(y ; K) \cap K \neq \varnothing$ then $K$ is said to be a co-sun, a notion which was introduced by P. L. Papini and I. Singer in connection with problems within the theory of best approximation, see L. Hetzelt [10] and U. Westphal [14] for details. With use of this notion, for an $m$-accretive operator on $X$ the closure of its domain is a co-sun.

We want to state a few facts about co-suns which seem to be of relevance in connection with accretive operators, see [9] for proofs.

(5.4) A subset in $\mathbf{R}^{n}$ is a co-sun exactly when it is closed and convex, and the metric projection onto it is the uniquely defined contractive retraction of $\mathbf{R}^{n}$ onto it (F.O. L. Klore).

(5.5) $A$ subset in the normed plane is a co-sun exactly when it is closed and $\|\cdot\|$-convex which in turn is the range of a contractive. ray retraction (L. A. Karlovitz, P. Gruber, L. Hetzelt).

In $1941 \mathrm{~F}$. Bohnenblust characterized those subspaces in $l_{n}^{p}, 1<p<$ $\infty, p \neq 2$ which are the ranges of contractive linear projectinos. He proved, a hyper-subspace is the range of a contractive linear projection exactly when its normal vector contains at most two nonzero coefficients, and concluded that a subspace has this property when and only when it can be written as the intersection of such hyper-subspaces. The second named author extended Bohnenblust's characterization as follows.

(5.6) Let $U_{n}$ denote the set of unit vectors in $\mathbf{R}^{n}$ which have at most two nonzero coefficients. $A$ subset in $l_{n}^{p}, 1<p<\infty, p \neq 2$, is a co-sun exactly when it is the intersection of a family of closed half spaces the normal vectors of which belong to $U_{n}$.

For $l_{n}^{1}$ and likewise for $l_{n}^{\infty}$ those subspaces which are the ranges of linear contractions have been characterized, but as far as we know there are no descriptions of co-suns for these spaces.

Also there is not much known about the ranges of $m$-accretive operators. 
If $X$ is an inner product space and $A$ a maximally monotone operator on $X$, so is $A^{-1}$ and, consequently, the closure of $R(A)$ is convex. This result has its counterpart for the normed plane.

(5.7) If $A$ is accretive on the normed plane, then, by Lemma 4.2, $A^{-1}$ is accretive with respect to the dual *norm. Consequently, if $A$ is $m$-accretive then $\overline{R(A)}$ is $\|\cdot\|^{*}$-convex.

Note added in proof. Professor S. Reich kindly pointed out to the authors that A. Cernès [Israel J. Math. 19 (1974), 335-48] already proved (4.2) even for $n$-spaces. In the plane he further verified that the two notions of accretiveness coincide exactly when the unit ball is either an ellipse or a parallelogram. In [J. Funct. Anal. 26 (1977), 378-95] S. Reich among others extended Cernès first statement to smooth spaces.

Finally, following Reich's ideas on approximating zeros it is not difficult to prove: Let $A$ be $m$-accretive on $X$,

$$
\forall y \in X \exists a \in \overline{R(A)} \ni 0 \leq\left\langle a-a^{\prime}, y-a\right\rangle_{s} \quad \forall a^{\prime} \in \overline{R(A)},
$$

i.e., $\overline{R(A)}$ is a sun in the setting of best approximation.

\section{REFERENCES}

[1] H. Berens and L. Hetzelt, Die metrische Struktur der Sonnen in $l_{\infty}(n)$, Aequationes Math., 27 (1984), 274-287.

[2] On maximally accretive operators in the plane, in Anniversary Volume on Approximation Theory and Functional Analysis, ed. by P. L. Butzer, R. L. Stens, and B. Sz.-Nagy, ISNM 65. Birkhäuser Verlag, Basel 1984, 109-116.

[3] H. Brezis, Opérateurs Maximaux Monotones, Notas de Matemática (50). North-Holland Publ. Co., Amsterdam, London, 1973.

[4] R. C. Bruck, Nonexpansive projections on subsets of Banach spaces, Pacific J. Math., 47 (1973), 341-355.

[5] H. Busemann, The Geometry of Geodesics, Academic Press, New York 1955.

[6] M. G. Crandall and T. M. Liggett, $A$ theorem and a counterexample in the theory of semigroups of nonlinear transformations, Trans. Amer. Math. Soc., 160 (1971), 263-278.

[7] H. Debrunner and P. Flor, Ein Erweiterungssatz für monotone Mengen, Arch. Math., 15 (1964), 445-447.

[8] A. Granas and J. W. Jaworowski, Some theorems on multi-valued mappings of subsets of the Euclidean space, Bull. Acad. Polon. Sci. Sér. Sci. Math. Astronom. Phys., 7, No. 5 (1959), 227-283.

[9] L. Hetzelt, Über die beste Coapproximation im $\mathbf{R}^{n}$, Dissertation U Erlangen-Nürnberg 1981.

[10] Acad. Sci. Hung., 45 (1-2) (1985), 53-68. 
[11] J. M. Lasry and R. Robert, Analyse nonlinéaire multivoque, Cahiers de Mathématiques de la Décision No. 7611, Université Paris IX, Dauphine 1980.

[12] G. J. Minty, Monotone (nonlinear) operators in Hilbert space, Duke Math. J., 29 (1962), 341-346.

[13] Amer. Math. Soc., 13 (1962), 11-12.

[14] U. Westphal, Über die Existenz- und Eindeutigkeitsmengen bei der besten Ko-Approximation, in Linear Spaces and Approximation, ed. by P. L. Butzer, E. Görlich and B. Sz.-Nagy, ISNM 60. Birkhäser Verlag Basel 1981, 225-264.

Received April 30, 1985.

UNIVERSITÄT ERLANGEN-NÜRNBERG

BISMARCKSTRASSE $1 \frac{1}{2}$

D-8520 ERLangen, W. Germany 



\title{
PACIFIC JOURNAL OF MATHEMATICS \\ EDITORS
}

\author{
V. S. VARADARAJAN \\ (Managing Editor) \\ University of California \\ Los Angeles, CA 90024 \\ HERBERT CLEMENS \\ University of Utah \\ Salt Lake City, UT 84112 \\ R. FINN \\ Stanford University \\ Stanford, CA 94305
}

\author{
HERMANN FLASCHKA \\ University of Arizona \\ Tucson, AZ 85721
}

RAMESh A. GANGOLLI

University of Washington Seattle, WA 98195

VAUGHAN F. R. JONES

University of California

Berkeley, CA 94720

ROBION KIRBY

University of California

Berkeley, CA 94720
C. C. MOORE

University of California

Berkeley, CA 94720

H. SAMELSON

Stanford University

Stanford, CA 94305

HAROLD STARK

University of California, San Diego

La Jolla, CA 92093

\section{ASSOCIATE EDITORS}

\author{
R. ARENS \\ E. F. BECKENBACH \\ B. H. NeUmanN \\ F. WOLF \\ K. YOSHIDA \\ (1906-1982)

\section{SUPPORTING INSTITUTIONS} \\ UNIVERSITY OF ARIZONA \\ UNIVERSITY OF BRITISH COLUMBIA \\ CALIFORNIA INSTITUTE OF TECHNOLOGY \\ UNIVERSITY OF CALIFORNIA \\ MONTANA STATE UNIVERSITY \\ UNIVERSITY OF NEVADA, RENO \\ NEW MEXICO STATE UNIVERSITY \\ OREGON STATE UNIVERSITY \\ UNIVERSITY OF OREGON \\ UNIVERSITY OF SOUTHERN CALIFORNIA \\ STANFORD UNIVERSITY \\ UNIVERSITY OF HAWAII \\ UNIVERSITY OF TOKYO \\ UNIVERSITY OF UTAH \\ WASHINGTON STATE UNIVERSITY \\ UNIVERSITY OF WASHINGTON
}

The Supporting Institutions listed above contribute to the cost of publication of this Journal, but they are not owners or publishers and have no responsibility for its content or policies.

Mathematical papers intended for publication in the Pacific Journal of Mathematics should be in typed form or offset-reproduced (not dittoed), double spaced with large margins. Please do not use built up fractions in the text of the manuscript. However, you may use them in the displayed equations. Underline Greek letters in red, German in green, and script in blue. The first paragraph must be capable of being used separately as a synopsis of the entire paper. In particular it should contain no bibliographic references. Please propose a heading for the odd numbered pages of less than 35 characters. Manuscripts, in triplicate, may be sent to any one of the editors. Please classify according to the scheme of Math. Reviews, Index to Vol. 39. Supply name and address of author to whom proofs should be sent. All other communications should be addressed to the managing editor, or Elaine Barth, University of California, Los Angeles, California 90024.

There are page-charges associated with articles appearing in the Pacific Journal of Mathematics. These charges are expected to be paid by the author's University, Government Agency or Company. If the author or authors do not have access to such Institutional support these charges are waived. Single authors will receive 50 free reprints; joint authors will receive a total of 100 free reprints. Additional copies may be obtained at cost in multiples of 50 .

The Pacific Journal of Mathematics is issued monthly as of January 1966. Regular subscription rate: $\$ 190.00$ a year (5 Vols., 10 issues). Special rate: $\$ 95.00$ a year to individual members of supporting institutions.

Subscriptions, orders for numbers issued in the last three calendar years, and changes of address should be sent to Pacific Journal of Mathematics, P.O. Box 969, Carmel Valley, CA 93924, U.S.A. Old back numbers obtainable from Kraus Periodicals Co., Route 100, Millwood, NY 10546.

The Pacific Journal of Mathematics at P.O. Box 969, Carmel Valley, CA 93924 (ISSN 0030-8730) publish'es 5 volumes per year. Application to mail at Second-class postage rates is pending at Carmel Valley, California, and additional mailing offices. Postmaster: send address changes to Pacific Journal of Mathematics, P.O. Box 969, Carmel Valley, CA 93924.

PUBLISHED BY PACIFIC JOURNAL OF MATHEMATICS, A NON-PROFIT CORPORATION

Copyright (C) 1986 by Pacific Journal of Mathematics 


\section{Pacific Journal of Mathematics}

\section{Vol. 125, No. $2 \quad$ October, 1986}

Dale Edward Alspach, On $\mathscr{L}_{p, \lambda}$ spaces for small $\lambda \ldots \ldots \ldots \ldots \ldots \ldots 257$

Jong Sook Bae and Sangsuk Yie, Range of Gateaux differentiable operators

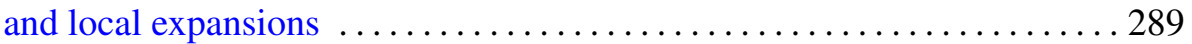

Hubert Berens and Lothar Hetzelt, On accretive operators on $l_{n}^{\infty} \ldots \ldots 301$

Marie-Françoise Bidaut-Véron, Global existence and uniqueness results for singular solutions of the capillarity equation ................. 317

Donald M. Davis and Mark Mahowald, Classification of the stable homotopy types of stunted real projective spaces ................. 335

Aad Dijksma, Heinz K. Langer and Hendrik S. V. de Snoo, Unitary

colligations in $\Pi_{\kappa}$-spaces, characteristic functions and Štraus extensions

Michel Enock and Jean-Marie Schwartz, Algèbres de Kac

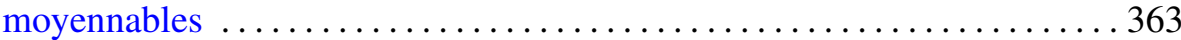

Seppo Granlund, Peter Lindqvist and Olli Martio, Note on the

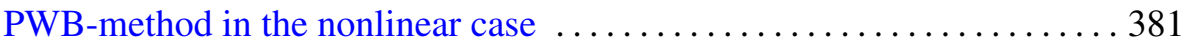

Palle E. T. Jorgensen, Analytic continuation of local representations of Lie

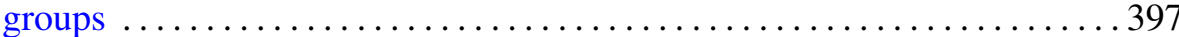

Robert P. Kaufman, Plane curves and removable sets ................409

José M. Montesinos and Wilbur Carrington Whitten, Constructions of two-fold branched covering spaces $\ldots \ldots \ldots \ldots \ldots$.

Benedict Seifert, Highly transitive group actions on trees and normalizing Tits systems

Charles Stuart Stanton, Counting functions and majorization for Jensen

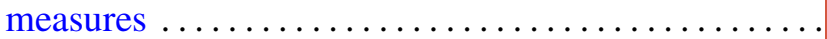

Luen-Fai Tam, On existence criteria for capillary free surfaces without gravity

Zhuocheng Yang, Exposed points of left invariant means 